\title{
RESULTS AFTER PARTIAL LEFT VENTRICULECTOMY VERSUS HEART TRANSPLANTATION FOR IDIOPATHIC CARDIOMYOPATHY
}

Steven W. Etoch, MD

Steven C. Koenig, PhD

Mary Ann Laureano, RN

Pat Cerrito, $\mathrm{PhD}$

Laman A. Gray, MD

Robert D. Dowling, MD
Objective: Partial left ventriculectomy has been introduced as an alternative surgical therapy to heart transplantation. We performed a singlecenter, retrospective analysis of all patients with idiopathic dilated cardiomyopathy who underwent partial left ventriculectomy or heart transplantation or who were listed for transplantation to determine operative mortality rate, 12-month survival, freedom from death on the heart transplantation waiting list, and freedom from death or need for relisting for heart transplantation. Methods: Patients who had partial left ventriculectomy (October 1996 to April 1998) were retrospectively compared with patients who were listed for heart transplantation (January 1995 to April 1998). Survival was assessed after the surgical procedure (partial left ventriculectomy vs heart transplantation) and from time of listing for heart transplantation to assess the additional impact of waiting list deaths. Freedom from death or relisting for heart transplantation was also compared. Results: There was no difference in age or United Network for Organ Sharing status between the 2 groups. Twenty-nine patients with idiopathic dilated cardiomyopathy were listed for heart transplantation; 17 patients underwent transplantation, 6 patients died while on the waiting list, and 6 patients remain listed. One patient died after heart transplantation, and 1 patient required relisting. Sixteen patients had partial left ventriculectomy; 10 patients are in improved condition, 2 patients died (1 death early from sepsis and 1 death from progressive heart failure), and 4 patients required relisting for heart transplantation. Operative survival was $94 \%$ after partial left ventriculectomy and 94\% after heart transplantation $(P=.92)$. Postoperative 12-month Kaplan-Meier survival was $86 \%$ after partial left ventriculectomy and $93 \%$ after heart transplantation $(P=.90)$. Twelvemonth Kaplan-Meier survival after listing for heart transplantation was 75\% due to death while on the waiting list $(P=.76)$. Freedom from death or need for relisting for heart transplantation was $56 \%$ after partial left ventriculectomy and $86 \%$ after transplantation $(P=.063)$. Conclusion: Operative and 12-month survival after partial left ventriculectomy and heart transplantation were comparable. However, despite their initial improvement, many patients who underwent partial left ventriculectomy required relisting for transplantation. Although partial left ventriculectomy is associated with acceptable operative and 12-month survival, it may prove to serve better as a bridge to transplantation in patients with idiopathic dilated cardiomyopathy rather than definitive therapy, given the number of patients who required relisting for transplantation. (J Thorac Cardiovasc Surg 1999;117:952-9)
From the Department of Surgery, Division of Cardiothoracic Surgery, University of Louisville, Louisville, Ky.

Read at the Twenty-fourth Annual Meeting of The Western Thoracic Surgical Association, Whistler, British Columbia, June 24-27, 1998.

Received for publication July 15, 1998; revisions requested Aug 17, 1998; revisions received Jan 12, 1999; accepted for publication Jan 13, 1999

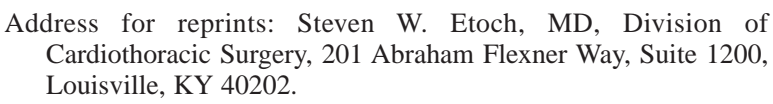

Address for reprints: Steven W. Etoch, MD, Division of Cardiothoracic Surgery, 201 Abraham Flexner Way, Suite 1200, Louisville, KY 40202.

Copyright $@ 1999$ by Mosby, Inc.

$0022-5223 / 99 \$ 8.00+0 \quad \mathbf{1 2 / 6 / 9 7 2 2 7}$ 
O thotopic heart transplantation (OHT) remains the standard with which all other alternative surgical therapies for the treatment of end-stage cardiomyopathies are compared. Today with the current practices of immunosuppression and the use of surveillance endomyocardial biopsy, survival after OHT is $84 \%$ at 1 year and $74.5 \%$ at 3 years in the United States. ${ }^{1}$ Despite these appealing results, only a small percentage of patients who could potentially benefit will receive OHT because of the shortage of donor supply, the strict selection criteria, and the substantial financial considerations. Recently, partial left ventriculectomy (PLV) was introduced by R. J. V. Batista, MD, of Curitiba, Brazil, as an alternative surgical therapy for the treatment of patients with end-stage dilated cardiomyopathy. To date, little is known about the potential benefits or the long-term results of this procedure. We therefore performed a single-center, retrospective analysis of all patients with idiopathic dilated cardiomyopathy (IDCM) who underwent OHT or PLV to determine operative mortality rates, 12-month survival, and freedom from death or need for relisting for OHT.

\section{Methods}

From October 1996 to April 1998, all patients evaluated with end-stage heart failure as the result of IDCM were considered potential candidates for PLV. All of these patients underwent a complete heart transplantation evaluation, which included exercise oxygen consumption, dobutamine echocardiography, and quality of life questionnaire. Patients with IDCM, left ventricular end-diastolic diameter (LVEDD) greater than $7 \mathrm{~cm}$, New York Heart Association (NYHA) class IV symptoms despite maximal medical therapy, and severely impaired exercise oxygen consumption were considered candidates for PLV. Patients were not excluded from PLV on the basis of right ventricular dysfunction, elevated transpulmonary gradient, presence of incidental coronary disease, need for inotropic agents, or an intra-aortic balloon pump. Patients who met all criteria for both OHT and PLV were given both options. Patients who did not meet the requirements for transplantation were given the option of PLV or continued maximal medical management. This study was approved by the Institutional Review Boards of the University of Louisville and Jewish Hospital, Louisville, Kentucky. All of the follow-up studies were performed at Jewish Hospital, Louisville, Kentucky. ${ }^{3}$

Patients who had PLV were retrospectively compared with all patients with IDCM who were listed for OHT from January 1995 to April 1998. Age, gender, United Network for Organ Sharing (UNOS) status, and death were identified for all patients. The primary objective was to compare postoperative survival between patients who had PLV or OHT. The Kaplan-Meier method was used to estimate postoperative survival for the PLV and OHT groups. ${ }^{2}$ Log-rank statistics and
Cox regression were used to compare the postoperative PLV and OHT groups statistically (SAS Institute, Cary NC), where the initial time ( $\mathrm{t}=0$ days) was defined as the date of the procedure (PLV or OHT). A secondary objective was to compare postoperative survival after PLV with survival from the time of listing for OHT. The Kaplan-Meier method was used to estimate survival. ${ }^{2}$ Log-rank statistics and Cox regression were used to compare the PLV and OHT groups, where the initial time ( $t=0$ days) was defined as the date of the procedure (PLV) and the date of listing for OHT. A third objective was to compare postoperative survival and freedom from relisting for OHT between patients who had a PLV or OHT surgical procedure. The Kaplan-Meier method was used to estimate postoperative survival and freedom from relisting rates for the PLV and OHT groups. ${ }^{2}$ Log-rank statistics and Cox regression were used to compare the postoperative survival and freedom from relisting for OHT for the PLV and OHT groups, where the initial time $(\mathrm{t}=0$ days $)$ was defined as the date of the procedure (PLV or OHT).

Our operative technique for PLV has previously been described and is reviewed here for completeness. ${ }^{3}$ Echocardiographic assessment of LVEDD was determined before the operation with a transgastric short axis view for assessment of the interpapillary muscle distance to determine whether resection of the lateral wall between the papillary muscles would be adequate to decrease the LVEDD to approximately $6 \mathrm{~cm}$ or less. If resection of the interpapillary muscle would not result in adequate decrease in LVEDD, a decision was made to proceed with either mitral valve replacement or mitral valve repair with reimplantation of the papillary muscle. There were no strict guidelines for deciding between repair and replacement. However, if we planned to resect more than $3 \mathrm{~cm}$ posterior to the posterior papillary muscle, mitral valve replacement was performed through the left ventriculotomy. Tricuspid valve annuloplasty was performed for significant $(>2+)$ regurgitation.

A median sternotomy was performed with standard aortic and bicaval cannulation, and cardiopulmonary bypass was initiated. The tricuspid valve was repaired on the beating heart. For patients who required mitral valve repair, the mitral valve was exposed through a standard left atriotomy after arrest of the heart with antegrade and retrograde cardioplegia. Standard annuloplasty with a Physio ring (Baxter CarpentierEdwards; Baxter Health Care Corp, Irvine Calif) was performed by downsizing the ring by 1 or 2 sizes to ensure adequate leaflet coaptation and thereby prevent central regurgitation. After completion of the mitral valve repair, a warm dose of cardioplegic solution was given, and the aortic crossclamp was released. An incision was then made in the left ventricle to the left of the left anterior descending artery and $3 \mathrm{~cm}$ proximal to the apex. The position of the papillary muscles was determined, and an incision was extended along the base of the anterior papillary muscle to within $2 \mathrm{~cm}$ of the atrioventricular groove. The incision was then extended parallel to the left anterior descending artery and along the base of the posterior papillary muscle to complete the resection. Initially, the ventricle was closed with interrupted 2-0 sutures 


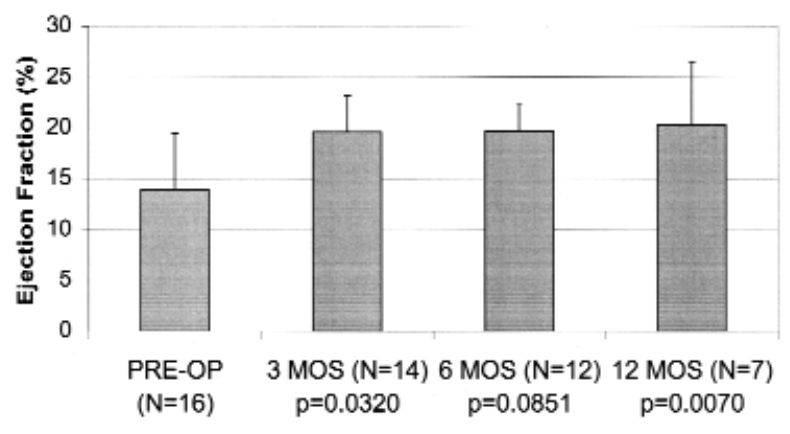

Fig 1. Improvement in left ventricular ejection fraction after PLV.

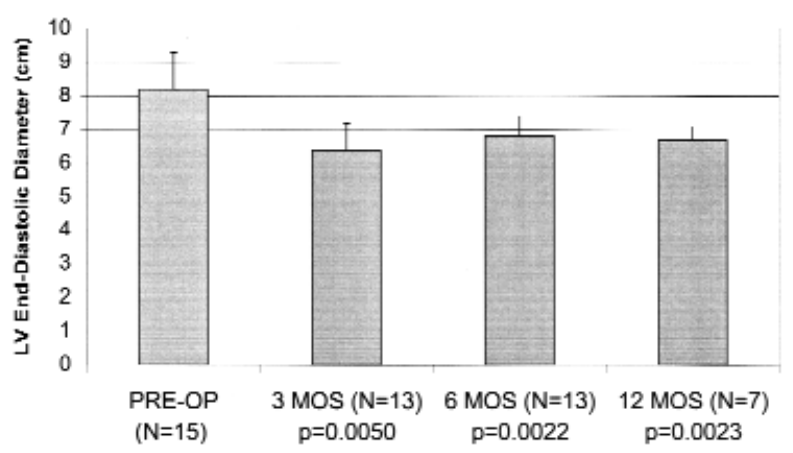

Fig 2. Reduction in LVEDD after PLV.

(Ethibond; Ethicon, Inc, Somerville, NJ) and felt strips followed by a 1-0 Ethibond running suture. Recently, we have closed the ventricle with 2 running sutures of 1-0 Vicryl polyglactin 910 (Ethicon). Aggressive deairing maneuvers were performed with echocardiographic evaluation for the presence of intracavitary air. The patients were then weaned from cardiopulmonary bypass. Postoperative echocardiographic assessment of mitral regurgitation, tricuspid regurgitation, and left ventricular function was performed. ${ }^{3}$

\section{Results}

From October 1996 to April 1998, 16 patients underwent PLV, with a mean follow-up of 11.1 months. Mean age was $49.6 \pm 10.5$ years (range, 30-67 years); 14 patients were male (14 of 16 patients, 88\%). All patients were in NYHA class IV heart failure and mean preoperative maximal exercise oxygen consumption was $13.5 \pm 3.0 \mathrm{~mL}$ oxygen per kilogram per minute. Six patients (6 of 16 patients, 37\%) were UNOS status I. Fifteen patients had mitral regurgitation with a mean grade of $3.3 \pm 0.7$. Thirteen patients had mitral valve repair, 2 patients had mitral valve replacement, and 1 patient without mitral regurgitation underwent PLV without a mitral valve procedure. Six patients required tricuspid valve repair.

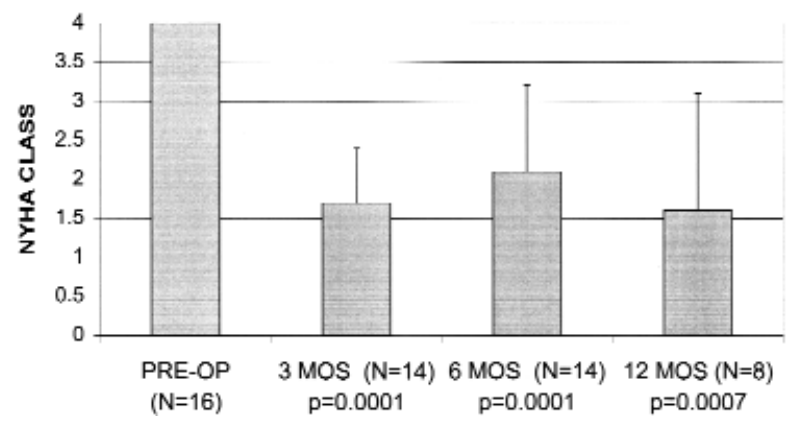

Fig 3. Improvement in NYHA class after PLV.

Table I. Comparison of PLV and OHT demographics

\begin{tabular}{lll}
\hline & \multicolumn{1}{c}{ PLV } & \multicolumn{1}{c}{ OHT } \\
\hline Patients (n) & 16 & 17 \\
Mean follow-up (mo) & 11.1 & $16.4^{*}$ \\
Mean age (y) & $49.6 \pm 10.5$ & $50.6 \pm 1.6^{\dagger}$ \\
Male:female (\%) & $14(88): 2(12)$ & $9(53): 8(47)^{\ddagger}$ \\
UNOS status I (\%) & $6(37)$ & $7(41)^{\S}$ \\
\hline
\end{tabular}

${ }^{*} P=.001$.

${ }^{\dagger} P=.328$.

${ }^{\ddagger} P=.031$.

${ }^{\S} P=.829$.

Left ventricular ejection fraction improved immediately after PLV from $13.9 \pm 5.6$ to $21.0 \pm 8.4(P=.001$, paired $t$ test) and continued to be significantly improved at the 3-month $(19.6 \pm 3.6)$, 6-month $(19.7 \pm 2.7)$, and 12-month $(20.3 \pm 6.2)$ postoperative follow-up examinaations (Fig $1 ; P=.032$, repeated-measures analysis of variance). LVEDD was significantly reduced after the operation, from $8.2 \pm 1.1$ to $6.4 \pm 0.8 \mathrm{~cm}(P=.002$, paired $t$ test), and continued to be significantly reduced at 3 months $(6.4 \pm 0.8 \mathrm{~cm}), 6$ months $(6.8 \pm 0.6 \mathrm{~cm})$, and 12 months $(6.7 \pm 0.4 \mathrm{~cm})$ after PLV (Fig 2; $P=.0001$, repeated-measures analysis of variance). The degree of mitral regurgitation was significantly decreased from $3.3 \pm 0.7$ before the operation to $0.1 \pm 0.0$ after the operation ( $P=.0004$, paired $t$ test). One patient had mild (1+) mitral regurgitation immediately after the operation, and 15 patients had no mitral regurgitation. Mitral regurgitation did not worsen in any of the patients during the 12-month follow-up period. Compared with preoperative values, there was a significant improvement in NYHA class at 3 months $(1.7 \pm 0.7), 6$ months $(2.1 \pm$ $1.1)$, and 12 months $(1.6 \pm 1.5$; Fig $3 ; P=.043$, repeated-measures analysis of variance).

There was 1 early death from an unknown cause after PLV on postoperative day 20. This patient had marked leukocytosis, dyspnea, and hypothermia. He was given 


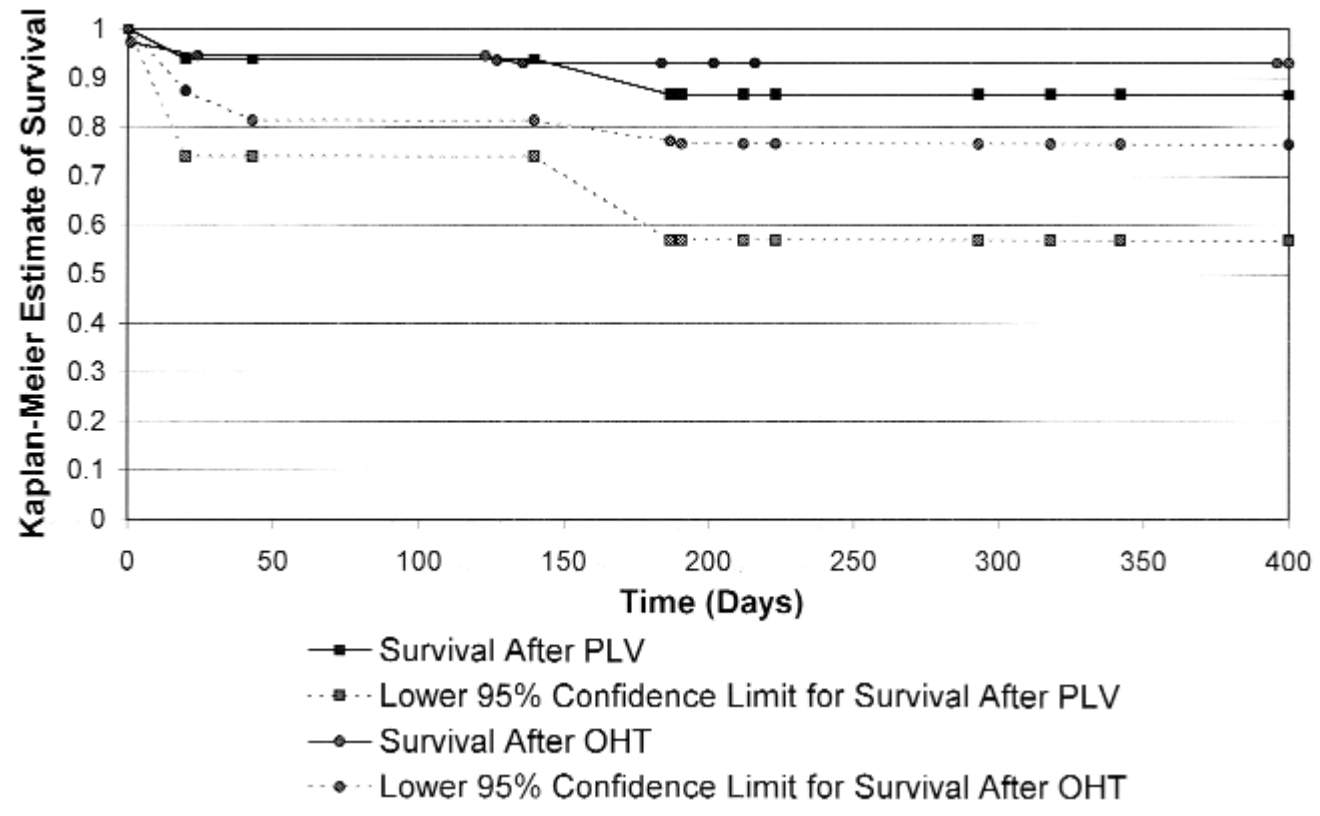

Fig 4. Survival after OHT and PLV.

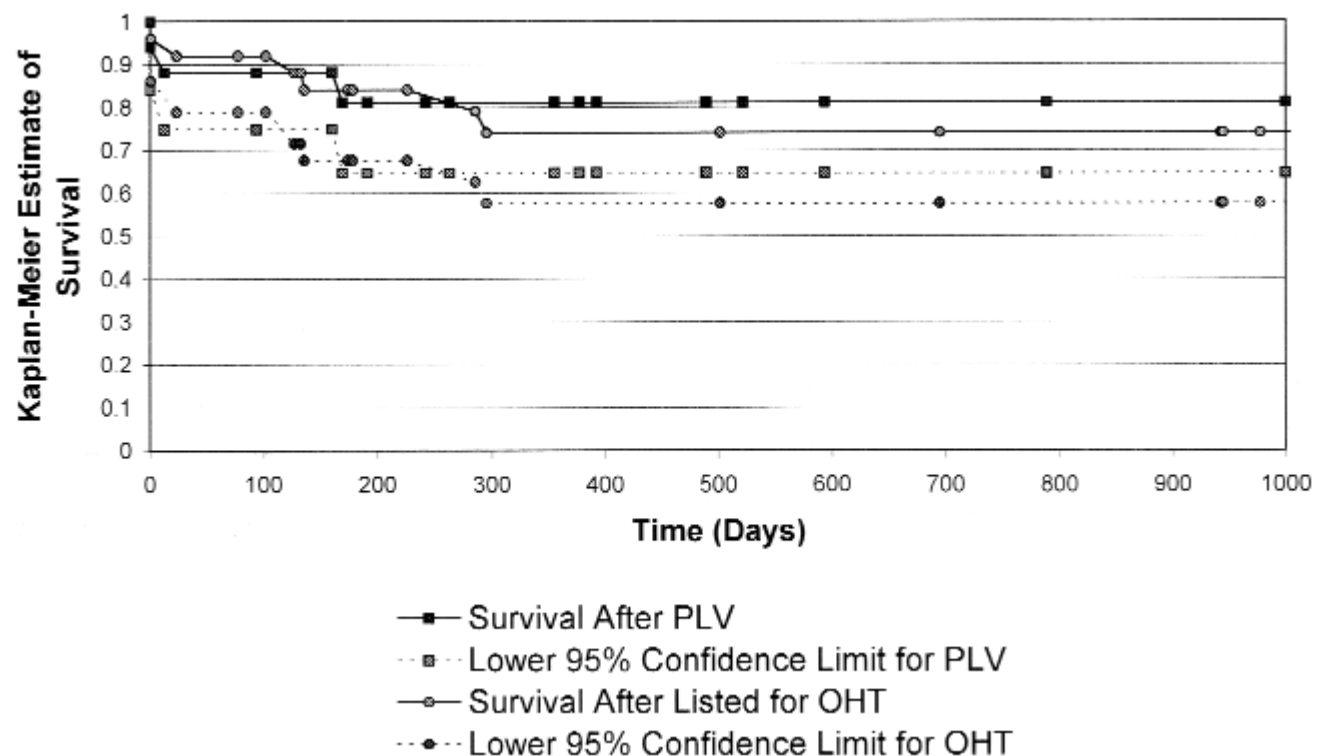

Fig 5. Survival after listing for OHT compared with survival after PLV.

broad-spectrum antibiotic therapy, but progressive hypotension and bradycardia developed. All efforts to resuscitate him were unsuccessful. An autopsy was not obtained.

Five patients who showed initial improvement in clinical status and left ventricle function after PLV experienced worsening heart failure. One of these patients died at 6 months after PLV as the result of worsening congestive heart failure and progressive multisystem organ failure. The other 4 patients required listing for transplantation on postoperative days $96,175,246$, and 348 . Two of these patients required placement of a left ventricular assist device on postoperative days 246 and 445. One patient placed on 


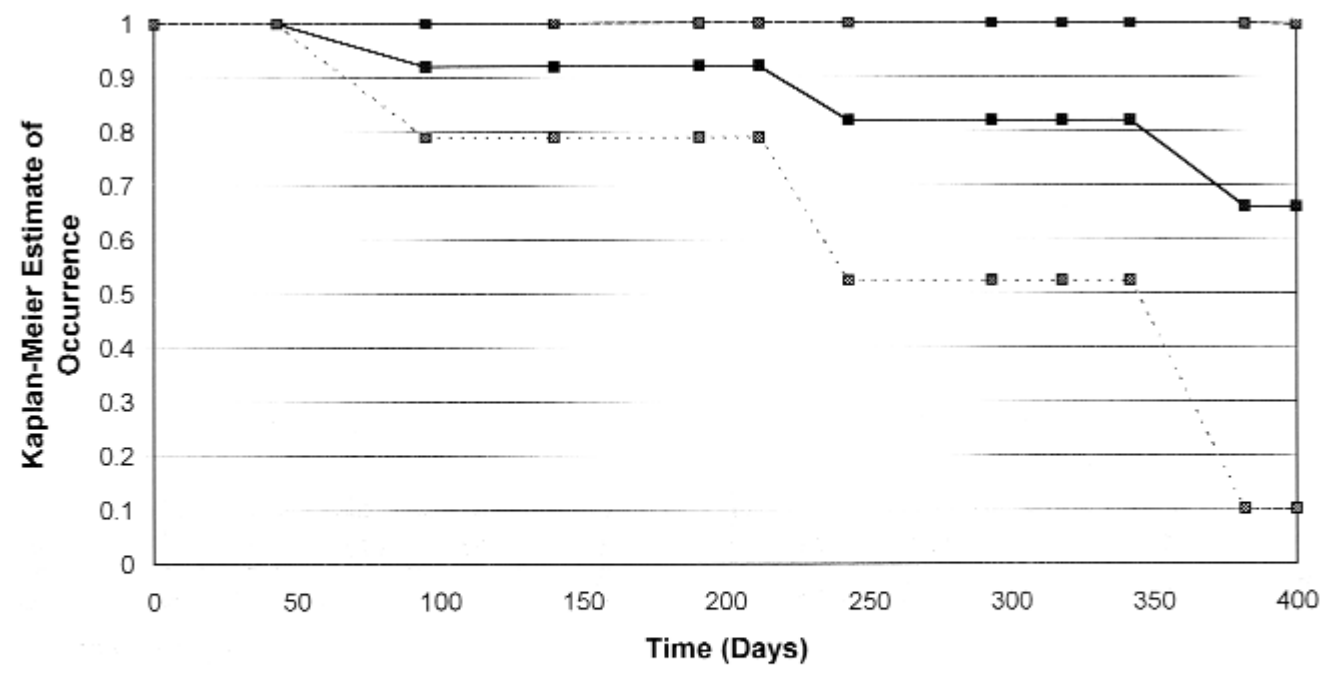

$\rightarrow$ Freedom From Listing for PLV

- - Lower 95\% Confidence Limit

-.. Upper 95\% Confidence Limit

Fig 6. Freedom from listing for OHT after PLV.

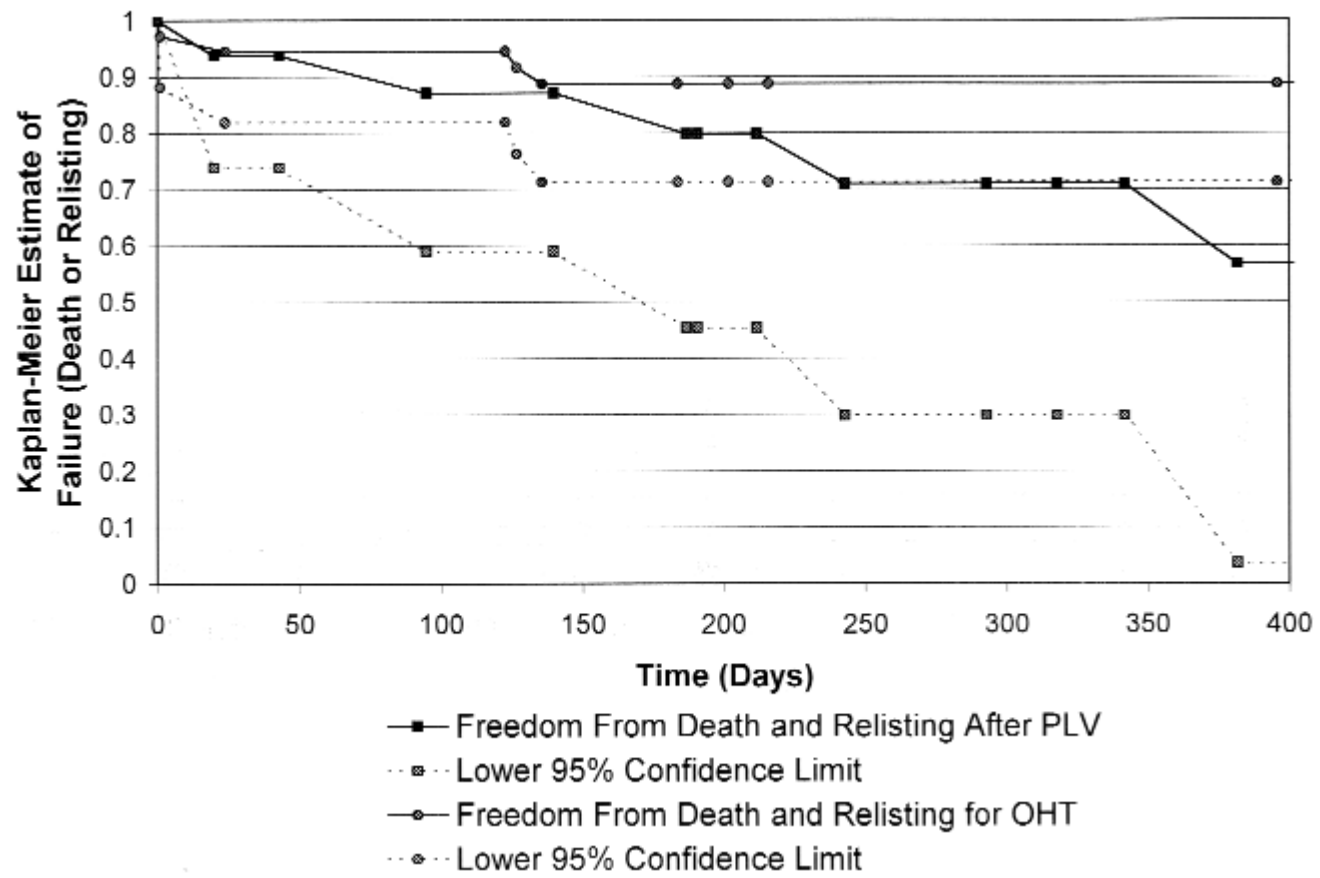

Fig 7. Freedom from death and relisting for OHT compared with PLV.

a left ventricular assist device has undergone transplantation and is doing well; the other 3 patients are awaiting transplantation.

From January 1995 to April 1998, 29 patients with
IDCM were listed for OHT. The average length of time on the waiting list before OHT was $140.2 \pm 60.8$ days (range, 3-595 days). Six of the 29 patients (21\%) died while on the waiting list, 6 patients remain on the wait- 
ing list, and 17 patients underwent OHT. The mean age of the OHT group was 50.6 \pm 1.6 years (range, 30-65 years), and there were 9 male patients ( 9 of 17 patients, $53 \%)$ compared with $88 \%$ in the PLV group $(P=.032)$.

The mean follow-up time was 16.4 months. Seven of the 17 patients $(41 \%)$ were UNOS status I compared with $37 \%$ in the PLV group $(P=.829$; Table I). One patient died 10 days after transplantation owing to multisystem organ failure. One patient was relisted for OHT as the result of refractory, chronic vascular rejection with subsequent graft dysfunction.

Operative mortality was $5.9 \%$ (1 of 17 patients), and 12-month Kaplan-Meier survival was $93 \%$ for OHT, compared with $6.3 \%$ (1 of 16 patients) and $86 \%$ for PLV (Fig 4). There was no significant difference in operative deaths $(P=.97)$ and 12 -month survival $(P=.90)$ between the PLV and OHT groups. Kaplan-Meier survival after listing for OHT (ie, after intent to treat) was $75 \%$ at 12 months, primarily the result of a $21 \%$ mortality rate while awaiting transplantation, compared with $86 \%$ survival after PLV $(P=.76$; Fig 5$)$. Freedom from need for relisting for OHT was $73 \%$ after PLV compared with $93 \%$ after OHT at 12 months $(P=.003$; Fig 6$)$. The Kaplan-Meier estimate of freedom from death or the need for relisting for OHT after PLV was $65 \%$ at 12 months compared with $86 \%$ after OHT ( $P=.063$; Fig 7).

\section{Discussion}

PLV was introduced by R. J. V. Batista as an alternative surgical treatment to improve left ventricular performance and to ameliorate the symptoms of heart failure in patients with end-stage cardiomyopathy. Batista and colleagues ${ }^{4}$ demonstrated that in some instances this operative approach would result in marked improvement in left ventricular performance and functional class. Recently, a number of centers have reported prospective trials of PLV with acceptable morbidity and mortality rates. ${ }^{5-10}$ However, there have been no studies to compare the results after PLV with the results seen after OHT. We therefore retrospectively reviewed our results after PLV and compared those to our results seen after OHT.

All patients in the PLV group and the OHT group had IDCM and met all standard medical criteria for heart transplantation. There was no significant difference between the age or UNOS status between the 2 groups; however, there was a difference in gender, with PLV being performed on predominantly male patients $(88 \%)$ whereas gender in patients having OHT was more evenly divided (53\%).

There was no difference in operative deaths between the 2 groups $(6.3 \%$ for the PLV group vs $5.9 \%$ for the OHT group; $P=.97)$. One early death after OHT was due to allograft dysfunction and multisystem organ failure. One early death occurred after PLV from presumed sepsis. There was also no significant difference in 12-month survival after PLV or OHT (86\% vs 93\%; $P=.90)$. However, 5 of the 16 patients who had PLV experienced worsening heart function. One of these patients ultimately died of progressive heart failure, and 4 of these patients have required relisting for transplantation. Two of these patients have required placement of a left ventricular assist device as a bridge to transplantation. This $31 \%$ incidence of worsening heart failure, with $13 \%$ requiring mechanical circulatory assistance, is consistent with findings published by others. ${ }^{9}$ However, unlike the experience reported by McCarthy and colleagues, ${ }^{9}$ our failures have usually occurred after discharge, with patients being relisted an average of 226 days after PLV. Conversely, only 1 patient required relisting for transplantation after OHT. This patient experienced refractory vascular rejection with subsequent biventricular failure.

Because of the $31 \%$ incidence of worsening heart failure after PLV, the freedom from relisting for heart transplantation at 12 months was 75\% after PLV compared with $94 \%$ after OHT. However, when considering the survival from time of intent to treat (time from listing for OHT vs time from PLV), the 12-month survival for the OHT group was $75 \%$ due to a waiting list mortality rate of $21 \%$. This compares with an $86 \%$ 12-month survival after PLV. Therefore the incidence of recurrent congestive heart failure after PLV has to be weighed against the presence of waiting list deaths in the OHT group.

In summary, the operative and 12-month survivals after PLV and OHT are comparable. However, despite their initial improvement, a number of patients who underwent PLV experienced recurrent heart failure, which resulted in death or required relisting for transplantation. Treatment with PLV seems to have the advantage of avoiding the significant risk of death while the patient is waiting for an appropriate donor organ. Although PLV is associated with acceptable operative and 12-month survival, it may prove to serve better as a bridge to transplantation in patients with IDCM rather than definitive therapy, given the number of patients who required relisting for transplantation. Our current efforts are focused on methods to identify the patients who are more likely to have a sustained improvement after PLV.

\section{REFERENCES}

1. Keck BM, Bennett LE, Fiol BS, Dally OP, Novick RJ, Hosenpud JD. Worldwide thoracic organ transplantation: a report from the UNOS/ISHLT International Registry for Thoracic Organ Transplantation. Clin Transpl 1996;31-45. 
2. Cantor A. Extending SAS survival analysis techniques for medical research. Cary [NC]: SAS Institute, Inc; 1997.

3. Dowling RD, Koenig S, Laureano MA, Cerrito P, Gray LA. Results of partial left ventriculectomy in patients with end-stage idiopathic dilated cardiomyopathy. J Heart Lung Tranplant 1998; 17:1208-12.

4. Batista RJV, Santos JLV, Takeshita N, Bocchino L, Lima PN, Cunha MA. Partial left ventriculectomy to improve left ventricular function in end-stage heart disease. J Card Surg 1996;11:96-7.

5. Batista RJV, Nery P, Bocchino L, et al. Partial left ventriculectomy to treat end-stage heart disease. Ann Thorac Surg 1997; 64:634-8.

6. Gorcsan J, Feldman AM, Kormos RL, Mandarino WA, Demetris AJ, Batista RJV. Heterogeneous immediate effects of partial left ventriculectomy on cardiac performance. Circulation 1998;97: 839-42.

7. Moreira LFP, Stolf NA, Bocchi EA, et al. Partial left ventriculectomy with mitral valve preservation in the treatment of patients with dilated cardiomyopathy. J Thorac Cardiovasc Surg 1998; 115:800-7.

8. Cury M, Higuchi L, Gutierrez PS, et al. Autopsy findings in early and late postoperative death of partial left ventriculectomy [abstract]. J Am Coll Cardiol 1998;31(suppl):225A.

9. McCarthy PM, Starling RC, Wong J, et al. Early results with partial left ventriculectomy. J Thorac Cardiovasc Surg 1997;114: 755-65.

10. Bocchi EA, Bellotti G, de Moraes AV, et al. Clinical outcome after left ventricular surgical remodeling in patients with idiopathic dilated cardiomyopathy referred for heart transplantation. Circulation 1997;96(Suppl):II165-72.

\section{Discussion}

Dr Steven Gundry (Loma Linda, Calif). The authors bring up a series of patients who were all potential transplantation candidates and who were qualified for transplantation, and they have compared these patients with a group of patients who had a Batista operation instead of the initial transplantation.

At our institution, we have used a Batista-type operation in 18 patients over the past 2 years. Specifically, we excluded transplantation candidates from this study. But it is interesting to note that after 24 months, our first 2 patients are still alive after a PLV. However, our experience overall has been that approximately one third of the patients will die, one third of the patients will be unchanged, and one third of the patients will improve. I notice the authors have come across very similar statistics, although phrased in a different way.

I have several questions for the authors. First, you have decided to compare different time periods in your study. Specifically, you have included an extra year and a half of looking at potential heart transplantation patients versus only approximately a year and a half total of Batista patients. Was there a reason that you went back farther to look at the heart transplantation candidates as opposed to the Batista patients?

Dr Etoch. Yes, we were trying to identify a very similar cohort for comparison and to compare the 2 groups from time of intent to treat. To achieve this we had to include the time on the waiting list for the heart transplantation group.
Dr Gundry. There would, of course, be a considerable difference in your outcome had the waiting list mortality rate for heart transplantation not been so large; that is, $21 \%$ of your patients on the heart transplantation list died while waiting. I am curious about the time period that you studied (January 1, 1995 , to the present). Were patients who were awaiting heart transplantation offered left ventricular assist devices when they became unstable?

Dr Etoch. Absolutely. We have available the Thoratec, ABIOMED, and Novacor left ventricular assist devices and are aggressive in using left ventricular assist devices as a bridge to transplantation.

Dr Gundry. Were these deaths that occurred while awaiting transplantation sudden deaths at home, or did they occur in the hospital, or do you know?

Dr Etoch. I do not know the exact numbers, but the majority were sudden deaths at home.

Dr Gundry. Am I correct that despite the Batista procedure there is really no difference in the waiting list or waiting probability of surviving for a heart transplantation candidate or a Batista candidate? Is that essentially what you are saying?

Dr Etoch. There was no difference in 1-year survival in patients treated with OHT or PLV.

Dr Gundry. Last, I noticed that all of these patients had typical dilated cardiomyopathy. Do you have any results of the Batista operation with ischemic ventricles?

Dr Etoch. No. As part of our prospective study of PLV, we tried to select a group that would be very homogeneous and be most likely to benefit from the procedure. For that reason, we specifically did not include patients with ischemic cardiomyopathy but specifically directed our attention to patients with IDCM.

Dr Gundry. I am impressed from your Batista results and a remarkable lack of late arrhythmic deaths. Our cardiologists have told us that if they were to design the perfect operation to create re-entrant ventricular tachycardia, they would design the Batista procedure. In fact all of our late deaths have been arrhythmic in nature. Many centers now routinely place an automatic implantable cardioverter defibrillator at the time of the initial operation for the Batista operation. Could you tell us the reason for the amazing lack of late arrhythmias in your series?

Dr Etoch. That has been a common problem presented by other centers as well, with ventricular arrhythmias resulting in a significant number of deaths. Unlike Dr Moreira and you, we have been very lucky that we have not had, to date, any sudden deaths attributable to ventricular arrhythmias after the operation. This is probably related to the fact that, because of the other data, we have routinely used amiodarone in our patients after PLV. We have also taken a very aggressive approach in placing automatic implantable cardioverter defibrillators both before and after the operation.

Dr Gundry. Finally, I noticed from your article and also from your slides that, despite an overall improvement in function even at 1 month after the Batista operation, by 6 and 12 months there begins to be a remarkably large increase in the standard deviation of the functional class in those patients. 
Does this imply that, despite an overall improvement in functional class, there are a large number of patients whose condition has begun to deteriorate, but who are hidden by the average number?

Dr Etoch. That is our impression as well, and the problem with this study and recent other series is the short follow-up period. If you look at our results, 5 of the 16 patients who underwent PLV, despite initial improvement, experienced worsening heart failure. For that reason, we concluded that with longer follow-up this may indeed ultimately prove to be a "biologic bridge" to transplantation as opposed to an alternative to transplantation.

Dr Gundry. Finally, what would you do for a patient who is going to require some form of intervention to get him to a heart transplantation? Would you try the Batista operation first, or would you go directly to an implantable left ventricular assist device?

Dr Etoch. As time has progressed, we have refined our approach to these patients, and we are currently looking at other indices that may suggest a better long-term result after PLV. Specifically, we are looking at the amount of myocardial fibrosis and myocyte diameter on endomyocardial biopsy and response with dobutamine echocardiography. We are less likely to advise patients with little improvement on dobutamine echocardiography, with myocyte diameter greater than
$30 \mathrm{~mm}$, and with significant fibrosis to undergo PLV. We currently consider patients who have improvement of left ventricular function on dobutamine echocardiography, have myocyte diameter less than $30 \mathrm{~mm}$, and have minimal myocardial fibrosis to be candidates for PLV.

Dr Marco Zenati (Pittsburgh, $\mathrm{Pa}$ ). As you are aware, the question of how to treat regurgitation in the face of partial ventriculectomy is the subject of much controversy. You also are aware of Dr Bowling's results, treating his patients exclusively with mitral valve repair or replacement without ventricular plasty. There are actually preliminary data that show, in fact, the degree of the residual mitral regurgitation as a predictor of rehabilitation and follow-up. Would you comment on how you handle mitral regurgitation?

Dr Etoch. As I mentioned, the mean grade of mitral regurgitation in our patient group was 3.3. Only 1 patient did not have mitral regurgitation before the operation; the remaining 15 patients all had significant mitral regurgitation and were treated at the time of the operation. Thirteen of these patients underwent mitral valve repair, and 2 of them underwent mitral valve replacement. Our 1 patient who did not have mitral regurgitation before the operation demonstrated similar results and improvements after the operation, despite requiring no treatment of her mitral valve.

\section{Online-www.aats.org}

Now you can get The Journal of Thoracic and Cardiovascular Surgery online. The Journal online brings you faster delivery time, easy searching of current and back issues, links to PubMed, AATS, WTSA and other important sites, and more. Visit the Journal online today. 